\title{
Visible-Light-Induced Deep Aerobic Oxidation of Alkyl Aromatics
}

\author{
Chang-Cheng Wang ${ }^{1}$, Guo-Xiang Zhang ${ }^{2}$, Zhi-Wei Zuo ${ }^{3^{*}}$, Rong Zeng ${ }^{2}$, Dan-Dan Zhai ${ }^{1}$, Feng Liu $^{1^{*}}$ \\ and Zhang-Jie Shi ${ }^{1,3^{*}}$. \\ ${ }^{1}$ Department of Chemistry, Fudan University. Shanghai, 200438, China. \\ ${ }^{2}$ Department of Chemistry, School of Science, Xi'an Key Laboratory of Sustainable Energy Materials Chemistry, Xi'an Jiaotong University, Xi'an, Shaanxi 710049, \\ China. \\ ${ }^{3}$ State Key Laboratory of Bioorganic and Natural Products Chemistry, Shanghai Institute of Organic Chemistry, Chinese Academy of Sciences, Shanghai 200032, \\ China. \\ ${ }^{4}$ State Key Laboratory of Organometallic Chemistry, Shanghai Institute of Organic Chemistry. Shanghai, 200032, China.
}

ABSTRACT: Oxidation is a major chemical process to produce oxygenated chemicals in both nature and the chemical industry. Currently, industrial deep oxidation processes from polyalkyl benzene are primary routes to produce benzoic acids and benzene polycarboxylic acids (BPCAs), while to some extent suffering from the energy-intensive and potentially hazardous drawbacks and the sluggish separation issues. In this report, visible-light-induced deep aerobic oxidation of (poly)alkyl benzene to benzene (poly)carboxylic acids was developed. $\mathrm{CeCl}_{3}$ was proved to be an efficient HAT (Hydrogen Atom Transfer)catalyst in the presence of alcohol as both hydrogen and electron shuttle. Dioxygen $\left(\mathrm{O}_{2}\right)$ was found as a sole terminal oxidant. In most cases, pure products were easily isolated by simple filtration, showing the advantages of scaling up. The reaction provides an ideal way to form valuable fine chemicals from abundant petroleum feedstocks.

Benzene polycarboxylic acids (BPCAs) are key intermediates in the preparations of resins, plasticizers, pharmaceutical acids, food preservatives, as well as the modulator in the synthesis of MetalOrganic Frameworks (MOF) ${ }^{1-6}$. Terephthalic acid (TPA), for example, be consumed principally as a monomer precursor in the manufacture of polyester (PET), has become one of the most indemand chemicals with an annual output of up to a hundred million tons $^{7}$. Benzoic acid, with million tons annually production, is commonly used as a $\mathrm{pH}$ adjustor, food preservation, and valuable starting material in the chemical industry ${ }^{8}$. Functionalized benzoic acids are also widely spread in drug discovery, material chemistry, agrochemicals as starting materials and critical ingredients. Salicylic acid itself is a druggable molecule and is mostly used in personal care, food \& preservatives. Mefenamic acid is known as a nonsteroidal anti-inflammatory drug (NSAID), which has beenused for short-term pain relief treatment and blood loss from menstrual periods (Fig.1a).

BPCAs are chemically synthesized through a series of oxidation reactions from the corresponding alkyl aromatics. The most notable commercial process, known as the Amoco process ${ }^{9}$, employs liquid-phase aerobic oxidation with homogeneous Co-Mn-Br catalyst system in acetic acid medium ${ }^{9-11}$. These processes commonly accompany high energy consumption at high temperatures $\left(175-225^{\circ} \mathrm{C}\right.$ ) and high dioxygen pressure (15-30 atm). The use of transition metal bromides ${ }^{12}$ leads to a potential hazard to the environment and stratospheric ozone. Indeed, several severe safety accidents were caused by this point in the past few years, which has triggered public panic and protest (Fig.1b) ${ }^{13}$. Other developed approaches in direct oxidations of alkyl aromatics include the following ways: i) stoichiometric oxidation by using highly reactive inorganic and/or organic oxidants $\left(\mathrm{KMnO}_{4}\right.$, iodine $\mathrm{V}$ species, $t$ - $\mathrm{BuOOH}, m$-CPBA, $\mathrm{H}_{2} \mathrm{O}_{2}$, NHPI, etc. $)^{14-20}$; ii) catalytic aerobic oxidation catalyzed by heterogeneous catalysts (e.g., polymer- or montmorillonite clay-supported catalysts, Pd, Au, or Co nanoparticles) (Fig.1b) ${ }^{10,21-24}$. The former processes suffered from the requirement of stoichiometric amounts of harmful or unsafe oxidants. The drawbacks of the latter protocols can be ascribed to some common disadvantages of heterogeneous catalysts, including the leaching problems of catalysts, limited oxidation efficiency and/or selectivity. Chemists never stop searching for a clean and efficient way for the catalytic oxidation of alkyl aromatics to BCPAs, to meet the green and sustainable requirements in industry. 
a)

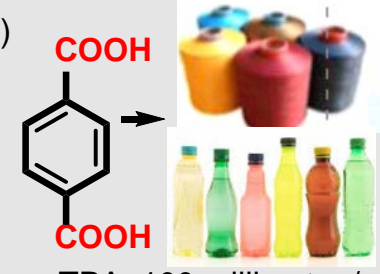

TPA, 100 million ton/a

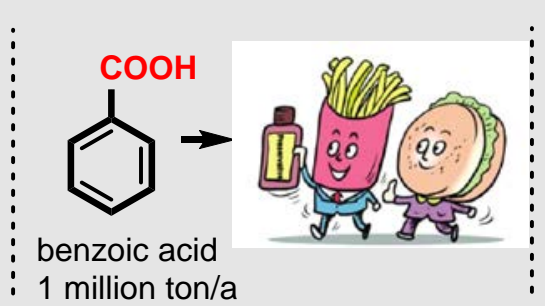

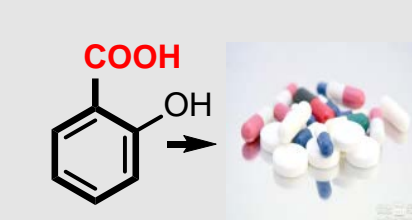

salicylic acid

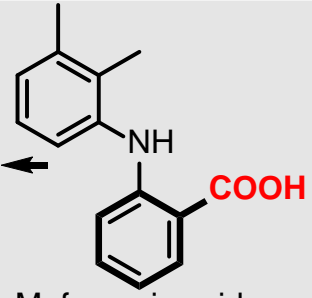

Mefenamic acid

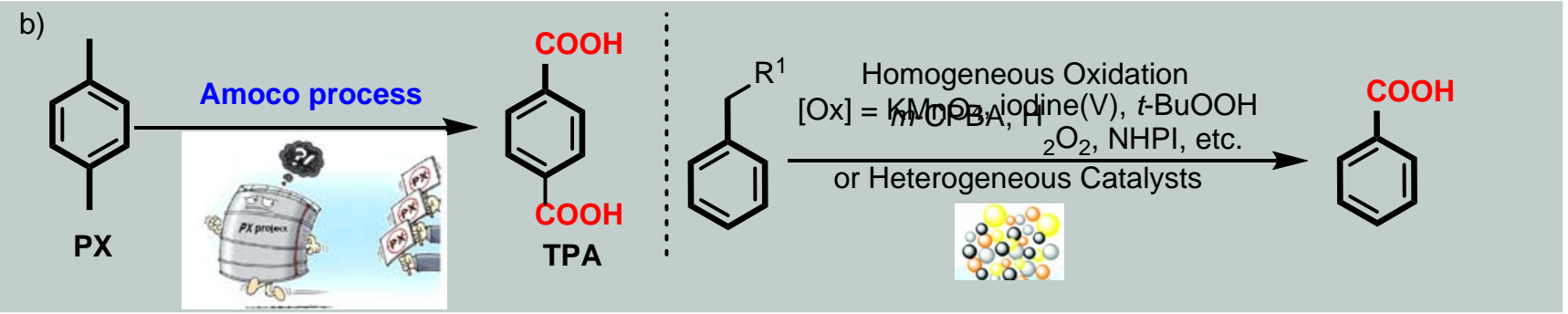

c)
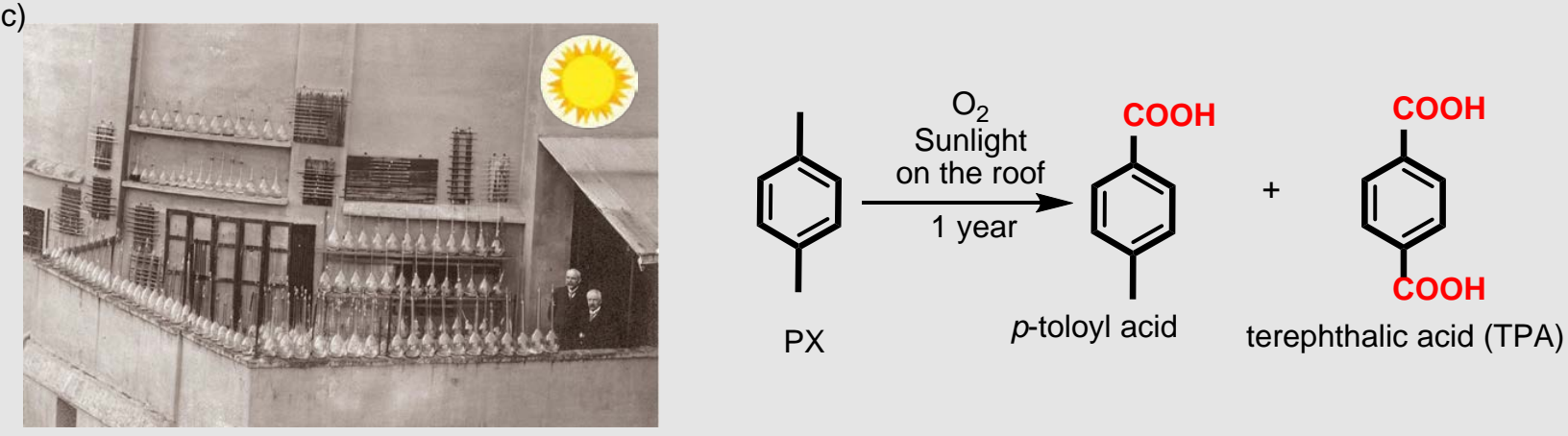

d)
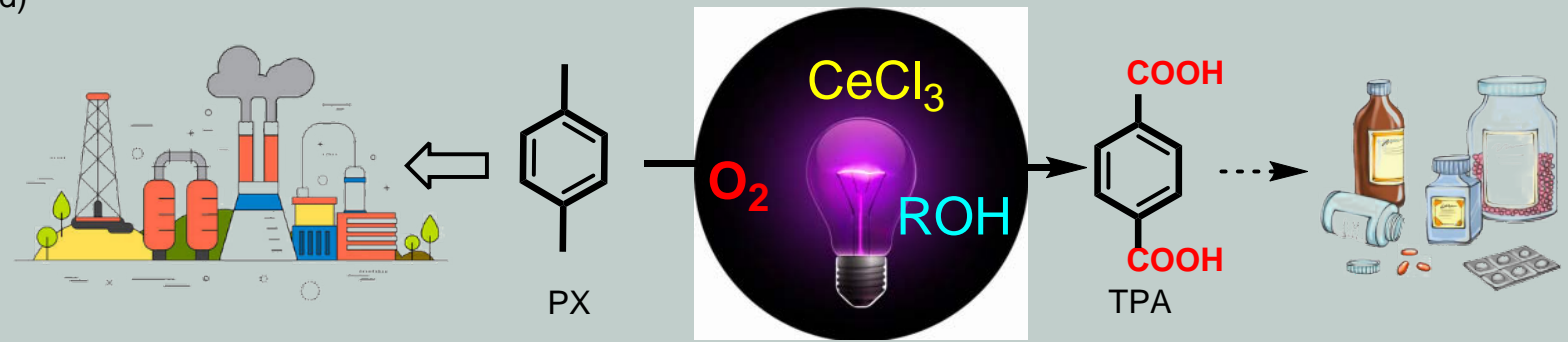

Fig.1 The nature of benzene (poly)carboxylic acids. a, Aromatic (poly)carboxylic acid-related products in daily life. b, Traditional oxidation of alkylbenzene. c, Photochemistry on the roof: The first oxidation of alkyl aromatics with molecular oxygen explored by Ciamician and Silber 100 years ago. d, Visible-light-induced deep aerobic oxidation of (poly)alkyl aromatics to aromatic (poly)carboxylic acids.

Ciamician and Silber reported the first photo-induced oxidation of alkyl aromatics with molecular oxygen in $1912^{25}$. Authors exposed toluene, o-xylene, m-xylene, $p$ xylene, and $p$-cymene to oxygen and sunlightfor about a year without any photosensitizer. The corresponding monocarboxylic acids were obtained as major products. Besides $m$ - and $p$-toluyl acids, iso and terephthalic acids were also formed from $m$ - and $p$-xylenes, respectively (Fig.1c). In recent years, photochemistry was re-announced, and photo-catalysts have been used to activate specific functional groups or substratesto complete various transformations via energy or electron transfer. This typical application was proved as a safe and green synthesis protocol in organic synthesis ${ }^{26-33}$. Several elegant aerobic C-H bond photo-oxidation protocols were developed towards the synthesis of alcohol, aldehyde, and ketones in the past few decades $^{34-39}$. Cerium salts were proved as efficient and favored photo-catalysts due to the relatively high abundance and excellent photo-catalytic performance. Zuo and coworkers developedsophisticated chemistry for the activation of $\mathrm{C}\left(s p^{3}\right)-\mathrm{H}$ bond through the synergistic utilization of hydrogen atom transfer (HAT) and ligand-to-metal charge 
transfer process (LMCT) with Ce catalysts ${ }^{40-41}$. By the analysis of recent developments, we envisioned that via a photo-induced, Ce-promoted benzylic $\mathrm{C}\left(s p^{3}\right)-\mathrm{H}$ bond activation, the toluene derivatives could be deeply oxidized tocarboxylic acids in the presence of molecular oxygen as terminal oxidant through the radical pathway (Fig.1d). This approach might present novel, green and sustainable oxidation in both fundamental sciences and chemical industry toward valuable fine chemical productions from abundant feedstocks.

\section{Results}

Reaction development. We set out to investigate the desirable photo-induced oxidation by examining 4-methyl1,1'-biphenyl 3n as a prototypical substrate. After careful optimization, we established an efficient photo-oxidation protocol using $\mathrm{CeCl}_{3}$ (5 mol\%) as a HAT catalyst in the presence of $\mathrm{CCl}_{3} \mathrm{CH}_{2} \mathrm{OH}$ (20 mol\% for mono-methyl benzenes, 1.0 equiv. for polymethyl benzenes), irradiated by LED light under $\mathrm{O}_{2}(1 \mathrm{~atm})$ in $\mathrm{CH}_{3} \mathrm{CN}$ for $24 \mathrm{~h}$ (see the supporting information for details.). The substrate scope was explored by oxidation of toluene and its derivatives. Benzoic acid 2a was obtained with $84 \%$ yield from toluene under standard conditions. The three xylenes 1b-1d were then irradiated with $40 \mathrm{w}$ Kessil PR160L-390 nm light to give the corresponding phthalic acids in good yields. As to the oxidation of orthoxylene $\mathbf{1 b}$, the in-situ formed phthalic acid will be spontaneously dehydrated to $\boldsymbol{o}$-phthalic anhydride $\mathbf{2 b}$. While trimethyl-, tetramethyl-, pentamethyl-, and even hexamethyl- benzenes (1e-1l) were introduced to the developed catalytic photo-oxidation system, we were happy to find that all of these methyl groups were fully converted to benzene polycarboxylic acids (BPCAs, 2e-2l). By a simple work-up, all of these conversions were in good to excellent yields, showing the power of this oxidation protocol (Fig.2a). Due to the importance of BPCAs and the long-standing environmental and safety problems in their syntheses, this methodology provides a sustainable way to produce those basic chemicals that benefit for the human's livelihood and national economy.

We then proceeded to test the scope of substituted toluene derivatives to extend the applications (Fig.2b). The oxidation of 2-methoxy- and 4-methoxy toluene underwent smoothly to give the corresponding benzoic acids $\mathbf{4 a}$ and $\mathbf{4 b}$ in $65 \%$ and $78 \%$ yield. A variety of para-substituted toluene substrates were then examined. Phenolic ester skeletons have recurred in many pharmaceutical molecules and natural products. The method makes it possible to proceed with the late-stage structure modification of those important molecules (4c-4e). Both electron-donating groups, like tertbutyl (4f), phenyl (4n), and electron-withdrawing groups $\mathrm{CF}_{3}$ (4g), $\mathrm{NO}_{2}$ (4h), COOEt (4I), CN (4m), as well as halogens (4i-4k) could tolerate this oxidation well, leaving useful synthetic functionalities for further derivatization. Meta-substituted toluene derivatives were oxidized to various benzoic acids (4o-4q), and slightly higher yields were given compared to their corresponding ortho- or paraisomers. Moreover, multi-substituted toluene derivatives were investigated, and all the oxidations worked well, showing good functional groups tolerance and synthetic diversity (4s-4x). 


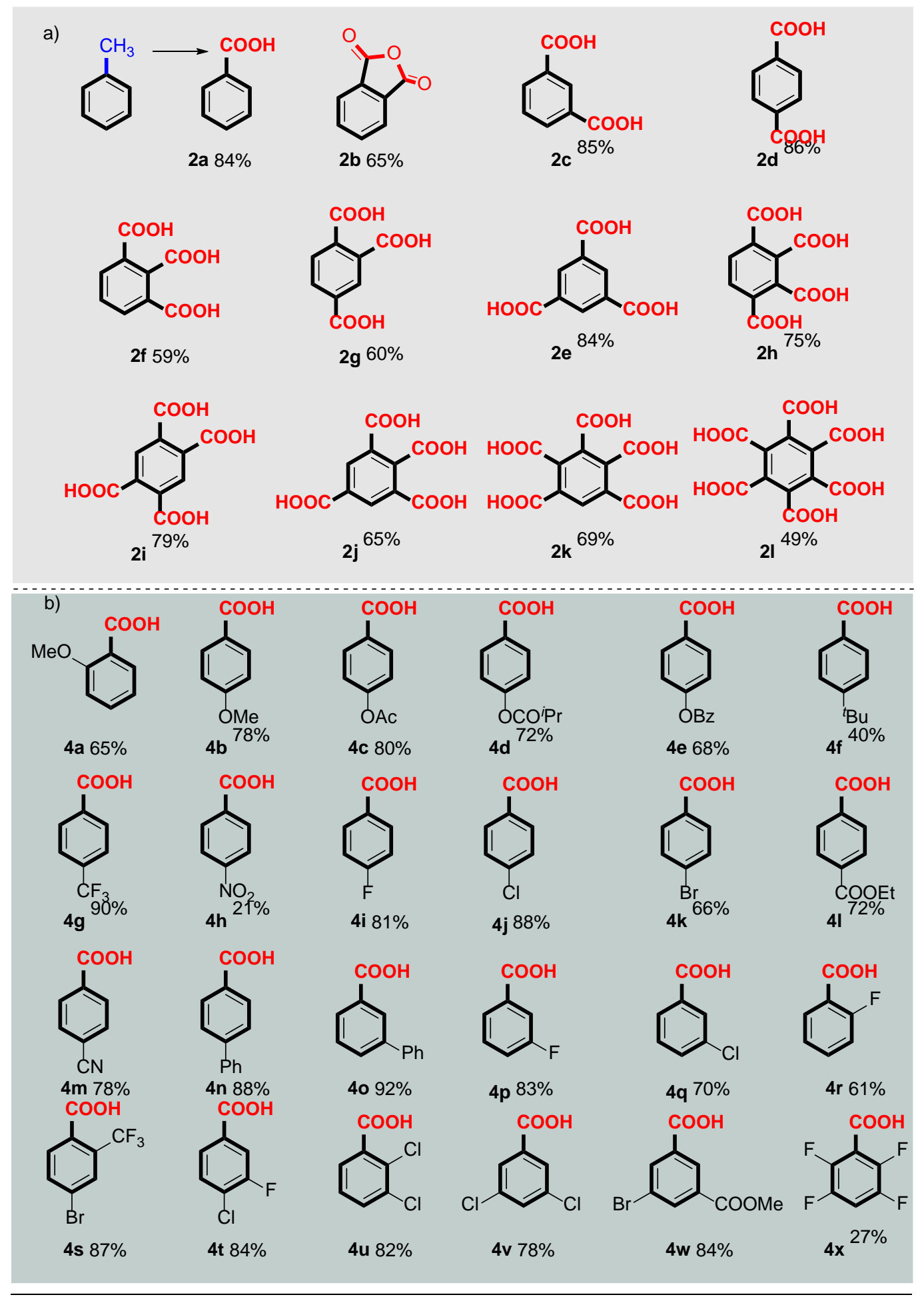

Fig.2 Deep areobic oxidation of (poly)methylbenzene. a, Oxidation of polyalkyl benzene $\mathbf{1}$ to BPCAs $\mathbf{2}$. b, Oxidation of substituted toluene $\mathbf{3}$ to substituted benzoic acids 4 . Conditions: 1 or $\mathbf{3}(0.1 \mathrm{mmol}), \mathrm{CeCl}_{3}(5.0 \mathrm{~mol} \%), \mathrm{CCl}_{3} \mathrm{CH}_{2} \mathrm{OH}(20 \mathrm{~mol} \%$ for mono-methyl benzenes, 1.0 equiv. for polymethyl benzenes) in $\mathrm{CH}_{3} \mathrm{CN}(2 \mathrm{~mL})$ under $1 \mathrm{~atm} \mathrm{O}_{2}$ atmosphere at $60{ }^{\circ} \mathrm{C}$, and irradiated with a $400 \mathrm{~nm} / 390 \mathrm{~nm}$ LED lamp for 24-72 h. See Supplementary Information for details.

Aromatic heterocyclic carboxylic acids widely existed in bio-active natural products, pharmaceutical compounds, and functional materials. Nevertheless, some could serve as multi-dentate ligands in coordination chemistry. We then introduced methyl heteroarenes to the developed oxidation system. To our delight, the oxidation reaction could proceed smoothly, given the target aromatic heterocyclic carboxylic acids with the moderate to good yields (Fig.3a). Thiophene2-carboxylic acid 6a, 5-acetyl thiophene-2-carboxylic acid 6b and benzo[b]thiophene-2-carboxylic acid $\mathbf{6 c}$ were obtained from the corresponding methyl- substrates $\mathbf{5 a - 5 c}$. 4-Methyl pyridine 5e and even 3-methyl-1-phenyl-1H- 
pyrazole 5f were compatible with this system, which could be converted to the heterocyclic carboxylic acid $\mathbf{6 e - 6 f}$, albeit the latter with a lower efficacy.

Substances with linear and branched alkyl substituents were then examined. We found that both benzylic C-H oxidation and C-C bond cleavage took place subsequently, delivering the corresponding carboxylic acid products in moderate yields (Fig.3b,3c). However, a benzylic C-H must be presented to initiate this oxidation process. It is important to note that when the mixture of toluene, ethylbenzene, cumene was introduced to this system, one sole benzoic acid product 2a was obtained with excellent yield (Fig.3d). This result illustrated that this oxidation reaction has a potent application in the separation and high-value transformation of the off-gases containing substituted benzene homologue compounds. Ultimately, this method was further applied to the late-stage modification of drugs. For example, the photocatalytic reaction of Celebrex, a non-steroidal antiinflammatory drug, delivered the oxidized product $\mathbf{1 0}$ in $65 \%$ yield in one step (Fig.3e). Considering the importance of this kind of medicinal agent, we expect this methodology to be useful for drug modification by introducing hydrophilic carboxylic acid groups at a very late stage.

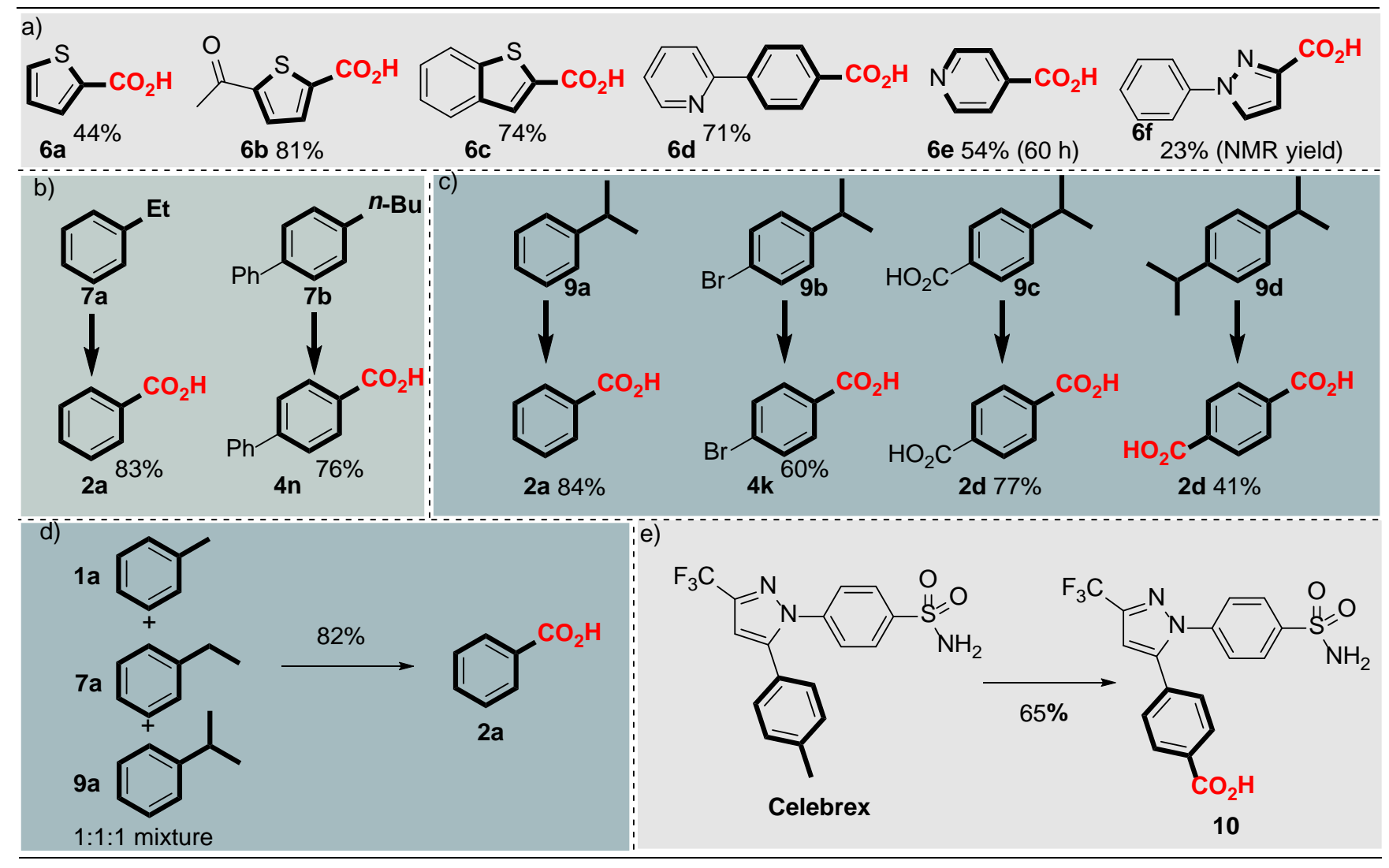

Fig. 3| The substrate extension of the oxidation. a, Oxidation of the benzylic C-H bond in heterocyclic compounds. b, Secondary benzylic C-H oxidation to benzoic acids through sequential cleavage of C-H and C-C bonds. c, Tertiary benzylic C-H oxidation. d, Oxidation of toluene, ethylbenzene, cumene mixture. e, Late-stage diversification of medicinal reagent under standard reaction conditions.

To explore the mechanism of this catalytic oxidation , a series of control experiments were conducted (Fig. 4a). Firstly, a radical scavenger (TEMPO) was added under standard conditions, and no targeted product $4 \mathbf{n}$ was detected, indicating a possible radical pathway for this oxidation reaction. While attempting to capture the intermediates with TEMPO in the absence of $\mathrm{O}_{2}$, compound $\mathbf{1 1}$ was detected by GC-MS. This result provided reliable support of the existence of benzylic radicals, which might be the initiator of this photo-induced radical oxidative procedure. ${ }^{18} \mathrm{O}_{2}$ was then used to take the place of $\mathrm{O}_{2}$, and undoubtedly only the desired ${ }^{18} \mathrm{O}$ labeled benzoic acid 4n' was obtained with good yield, which clearly illustrated that the [O] atom in the product came from $\mathrm{O}_{2}$. To get more insight on the reaction mechanism, we carried out the oxidation of $3 \mathbf{n}$ with $1.0 \mathrm{eq}$. of $\mathrm{CCl}_{3} \mathrm{CH}_{2} \mathrm{OH}$. The reaction was monitored by ${ }^{1} \mathrm{H}$ NMR spectroscopy every 15 minutes (Fig.4b). Two key intermediates were traced during the reaction. The hydroperoxide was first observed, it was further oxidized after the peak at $45 \mathrm{~min}$ (12, green volcano l). Aldehyde was produced and get to its summit at around 90 mins (13, red 
spot). Finally, both were transferred to the desired product

(4n, blue triangle) at 135 minutes.

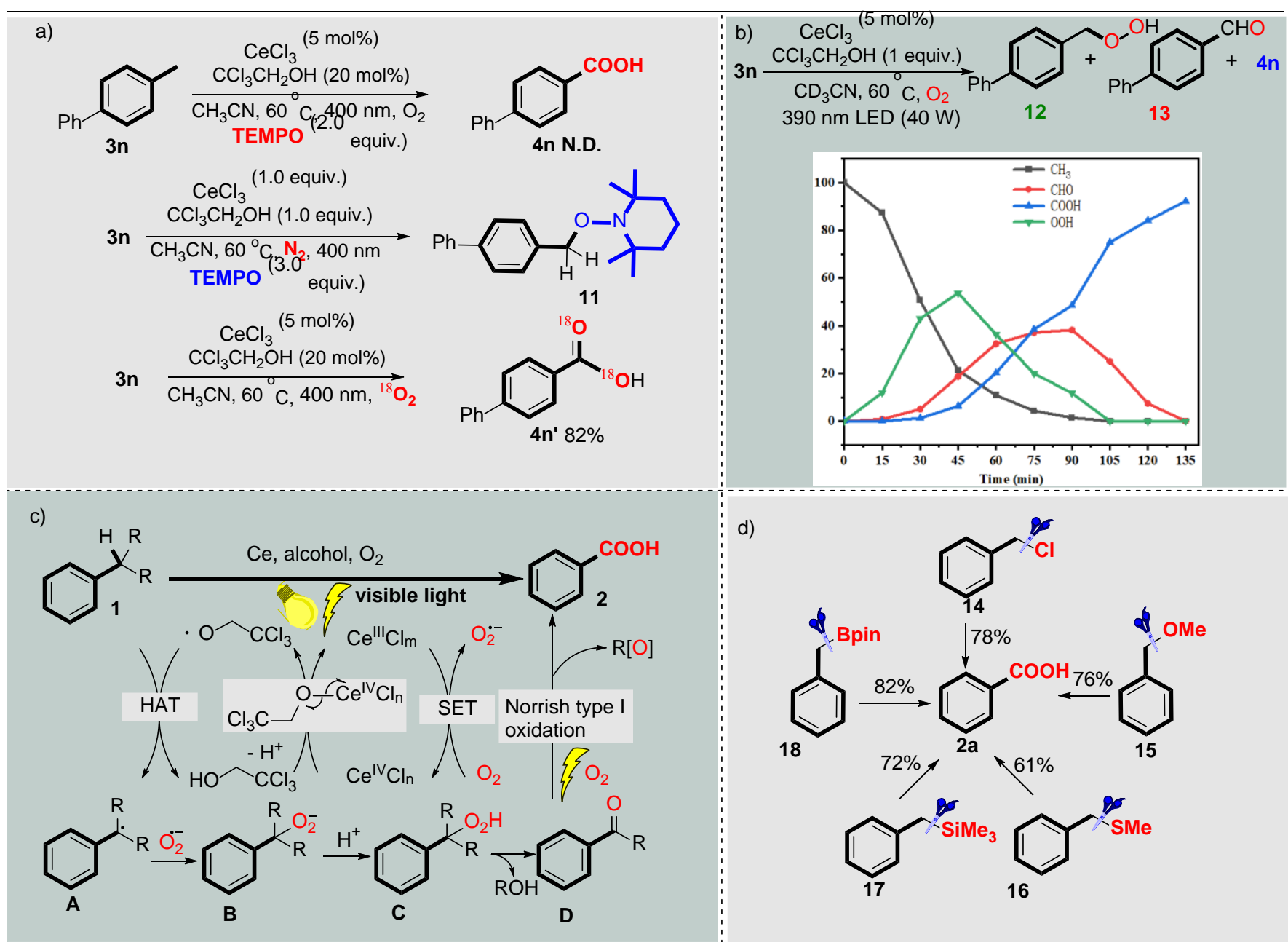

Fig.4| Mechanistic investigations. a, Control experiments. b, Kinetic experiments. c, Plausible mechanism for the oxidation from toluene to benzoic acids. d, The deep oxidation of benzyl derivatives with heteroatom group substituents.

Based on the observations and previous studies, a plausible mechanism was proposed in Fig.4c. First, electrophilic alkoxy radical was generated from alcohol via Ce (IV)-alkoxide intermediate participated photoinduced LMCT process. Alkoxy radical then abstracted the hydrogen from benzylic $\mathrm{C}-\mathrm{H}$ bond to form benzyl radical A; Subsequently, a single electron transfer occurred between molecular dioxygen $\left(\mathrm{O}_{2}\right)$ and $\mathrm{Ce}(\mathrm{III})$ to form superoxide radical; The in-situ formed superoxide radical was involved in the reaction and oxidized the alkyl radicals to hydroperoxide $\mathbf{B}$. After protonation and $\mathrm{ROH}$ released, D was obtained, which further accelerated the Norrish type I oxidation process to produce carboxylic acids 2 under illumination and heating conditions ${ }^{42}$. Under these reaction conditions, we further proved that both benzaldehyde and acetophenone could be further oxidized.

Understanding the feature of herein developed deep oxidation of toluene derivatives, we envisioned that different substituents at a benzylic position would not affect such oxidation. Therefore, we further investigated the oxidation reaction of benzyl derivatives with different functionalities (Fig.4d). To our delight, all tested hetero atom substituted toluene derivatives, including benzylic chloride 14, benzyloxy methane 15, benzyl(methyl)sulfane 16, benzyl-trimethyl silane 17, and benzylic borolane $\mathbf{1 8}$ was oxidized in this system as expected, and all gave benzoic acid $2 \mathbf{a}$ as the same sole product. These results detailed that both the C-H oxidation and the $\mathrm{C}-\mathrm{X}$ bond cleavage taken place under standard conditions. 
In conclusion, we have successfully developed a general and practical photocatalytic protocol for deep aerobic oxidation of (poly)alkylbenzene. To use the inexpensive and earth-abundant $\mathrm{CeCl}_{3}$ as the catalyst, $\mathrm{CCl}_{3} \mathrm{CH}_{2} \mathrm{OH}$ as HAT reagent, benzyl C-H, C-C bond, C$\mathrm{X}$ bonds were cleaved and finally oxidized to benzoic acid when irradiated with LED light under $\mathrm{O}_{2}$ atmosphere. The chemistry here showed good functional group tolerance and could be used as an efficient strategy in the late-stage modification in druggable molecules. Mechanistic studies unveiled the oxidation initiated from the hydrogen abstraction at the benzylic position. Thus the presence of benzyl C-H bond was the critical requirement to proceed this chemistry. Not only does this development provides a green and efficient protocol to produce the valuable substituted benzoic acids, heteroaromatic carboxylic acids and benzene polycarboxylic acids (BPCAs), but it offers new thinking to oxidize the aliphatic C-H bonds to oxygenated products from feedbacks of petroleum chemistry.

\section{AUTHOR INFORMATION}

\section{Corresponding Author}

zjshi@fudan.edu.cn, liufeng@fudan.edu.cn, zuozhw@shanghaitech.edu.cn

\section{ACKNOWLEDGMENT}

We thank the National Nature Science Foundation of China. This work was supported by NSFC (21988101, 21761132027, 22071029), Science and Technology Commission of Shanghai Municipality (19XD1400800, 18JC1411300), Shanghai Municipal Education Commission (2017-01-07-00-07-E00058), Key-Area Research and Development Program of Guangdong Province (2020B010188001), Shanghai Gaofeng \& Gaoyuan Project for University Academic Program Development, and the China Postdoctoral Science Foundation (2020M681144).

We thank Xue-Chen Luan (Fudan University) for help editing the manuscript.

\section{AUTHOR CONTRIBUTIONS}

S.Z-J. and L.F. directed the research and developed the concept of the reaction with W.C-C., who also performed the experiments and characterized all the products. Z.GX., Z.Z-W., Z.R., and Z.D-D. gave some helpful suggestions for the reaction and analyze the results . S.ZJ. and L.F. wrote the manuscript with contributions from the other authors.

\section{COMPETING INTERESTS}

The authors declare no competing interests.
Read, D. E. \& Purves, C. B. Separation of Benzene Hexa-, Penta- and the Three Isomeric Tetracarboxylic Acids and their Methyl Esters1. Journal of the American Chemical Society 74, 116-119, doi:10.1021/ja01121a027 (1952).

2 Holland, F. A. Kirk-Othmer encyclopedia of chemical technology, 3rd edition, vol. 1: Executive editor: Martin Grayson. Pp. 998. John Wiley, Chichester. 1978. £50.00. Endeavour 2, 193, doi:https://doi.org/10.1016/01609327(78)90109-6 (1978).

3 Windholz, M. The Merck Index Online. Science 226, 1250, doi:10.1126/science.226.4680.1250 (1984).

4 Berbrier, M. Making Minorities: Cultural Space, Stigma Transformation Frames, and the Categorical Status Claims of Deaf, Gay, and White Supremacist Activists in Late Twentieth Century America. Sociological Forum 17, 553591 (2002).

5 Kauffman, G. B. Ullmann's Encyclopedia of Industrial Chemistry Fifth Edition, First International Edition in English by Wolfgang Gehartz, Gail Schulz Thomas Kellersohn, Barbara Elvers, Stephen Hawkins, and Ulrike Winter (Eds.). The Chemical Educator 5, 49-53, doi:10.1007/s00897990360a (2000).

6 Stock, N. \& Biswas, S. Synthesis of MetalOrganic Frameworks (MOFs): Routes to Various MOF Topologies, Morphologies, and Composites. Chemical Reviews 112, 933-969, doi:10.1021/cr200304e (2012).

7 Luo, Z. W. \& Lee, S. Y. Biotransformation of pxylene into terephthalic acid by engineered Escherichia coli. Nature Communications 8, 15689, doi:10.1038/ncomms15689 (2017).

8 Lambert, R. J. \& Stratford, M. Weak-acid preservatives: modelling microbial inhibition and response. Journal of Applied Microbiology 86, 157-164, doi:https://doi.org/10.1046/j.13652672.1999.00646.x (1999).

9 Raghavendrachar, P. \& Ramachandran, S. Liquid-phase catalytic oxidation of p-xylene. Industrial \& Engineering Chemistry Research 31, 453-462, doi:10.1021/ie00002a001 (1992).

10 Ghiaci, M., Mostajeran, M. \& Gil, A. Synthesis and Characterization of Co-Mn Nanoparticles Immobilized on a Modified Bentonite and Its Application for Oxidation of p-Xylene to Terephthalic Acid. Industrial \& Engineering Chemistry Research 51, 15821-15831, doi:10.1021/ie3021939 (2012).

11 Tomás, R. A. F., Bordado, J. C. M. \& Gomes, J. F. P. p-Xylene Oxidation to Terephthalic Acid: A Literature Review Oriented toward Process Optimization and Development. Chemical 
Reviews 113, 7421-7469, doi:10.1021/cr300298j (2013).

12 Horst, A. et al. Stable bromine isotopic composition of methyl bromide released from plant matter. Geochimica et Cosmochimica Acta 125 , 186-195, doi:https://doi.org/10.1016/j.gca.2013.10.016 (2014).

13 Lee, K. \& Ho, M.-s. The Maoming Anti-PX Protest of 2014: An environmental movement in contemporary China. China Perspectives 3, 3339, doi:10.4000/chinaperspectives.6537 (2014).

14 Partenheimer, W. Methodology and scope of metal/bromide autoxidation of hydrocarbons. Catalysis Today 23, 69-158, doi:https://doi.org/10.1016/09205861(94)00138-R (1995).

15 Nammalwar, B., Fortenberry, C., Bunce, R. A., Lageshetty, S. K. \& Ausman, K. D. Efficient oxidation of arylmethylene compounds using nano-MnO2. Tetrahedron Letters 54, 2010-2013, doi:https://doi.org/10.1016/j.tetlet.2013.02.009 (2013).

16 Wang, F. \& Tong, Z. Dehydro-aromatization of cyclohexene-carboxylic acids by sulfuric acid: critical route for bio-based terephthalic acid synthesis. RSC Advances 4, 6314-6317, doi:10.1039/C3RA46670A (2014).

17 Baran, P. S. \& Zhong, Y.-L. Selective Oxidation at Carbon Adjacent to Aromatic Systems with IBX. Journal of the American Chemical Society 123, 3183-3185, doi:10.1021/ja004218x (2001). Hirai, N., Sawatari, N., Nakamura, N., Sakaguchi, S. \& Ishii, Y. Oxidation of Substituted Toluenes with Molecular Oxygen in the Presence of

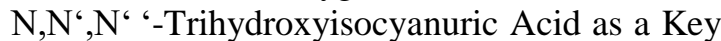
Catalyst. The Journal of Organic Chemistry 68, 6587-6590, doi:10.1021/jo034313z (2003).

19 Yavari, I. \& Karimi, E. N-HydroxyphthalimideCatalyzed Oxidative Production of Phthalic Acids from Xylenes Using O2/HNO3 in an Ionic Liquid. Synthetic Communications 39, 34203427, doi:10.1080/00397910902770461 (2009).

20 Zhang, J. et al. A metal-free catalytic system for the oxidation of benzylic methylenes and primary amines under solvent-free conditions. Green Chemistry 11, 1973-1978, doi:10.1039/B919346B (2009).

21 Kesavan, L. et al. Solvent-Free Oxidation of Primary Carbon-Hydrogen Bonds in Toluene Using Au-Pd Alloy Nanoparticles. Science 331, 195, doi:10.1126/science.1198458 (2011).

22 Sarma, B. B., Efremenko, I. \& Neumann, R. Oxygenation of Methylarenes to Benzaldehyde Derivatives by a Polyoxometalate Mediated Electron Transfer-Oxygen Transfer Reaction in
Aqueous Sulfuric Acid. Journal of the American Chemical Society 137, 5916-5922, doi:10.1021/jacs.5b01745 (2015).

23 Dhakshinamoorthy, A., Asiri, A. M., Herance, J. R. \& Garcia, H. Metal organic frameworks as solid promoters for aerobic autoxidations. Catalysis Today 306, 2-8, doi:https://doi.org/10.1016/j.cattod.2017.01.018 (2018).

24 Majumdar, B., Bhattacharya, T. \& Sarma, T. K. Gold Nanoparticle-Polydopamine-Reduced Graphene Oxide Ternary Nanocomposite as an Efficient Catalyst for Selective Oxidation of Benzylic C(sp3)-H Bonds Under Mild Conditions. ChemCatChem 8, 1825-1835, doi:https://doi.org/10.1002/cctc.201600136

(2016).

25 Ciamician, G. \& Silber, P. Chemische Lichtwirkungen. XXII. Autooxydationen. I. Berichte der deutschen chemischen Gesellschaft 45, 38-43, doi:https://doi.org/10.1002/cber.19120450107 (1912).

26 Narayanam, J. M. R. \& Stephenson, C. R. J. Visible light photoredox catalysis: applications in organic synthesis. Chemical Society Reviews 40, 102-113, doi:10.1039/B913880N (2011).

27 Prier, C. K., Rankic, D. A. \& MacMillan, D. W. C. Visible Light Photoredox Catalysis with Transition Metal Complexes: Applications in Organic Synthesis. Chemical Reviews 113, 53225363, doi:10.1021/cr300503r (2013).

28 Schultz, D. M. \& Yoon, T. P. Solar Synthesis: Prospects in Visible Light Photocatalysis. Science 343, 1239176, doi:10.1126/science.1239176 (2014).

29 Romero, N. A. \& Nicewicz, D. A. Organic Photoredox Catalysis. Chemical Reviews 116, 10075-10166, doi:10.1021/acs.chemrev.6b00057 (2016).

30 Yu, X.-Y., Chen, J.-R. \& Xiao, W.-J. Visible Light-Driven Radical-Mediated C-C Bond Cleavage/Functionalization in Organic Synthesis. Chemical Reviews, doi:10.1021/acs.chemrev.0c00030 (2020).

31 Shu, C., Noble, A. \& Aggarwal, V. K. Metal-free photoinduced C(sp3)-H borylation of alkanes. Nature 586, 714-719, doi:10.1038/s41586-0202831-6 (2020).

32 Pipal, R. W. et al. Metallaphotoredox aryl and alkyl radiomethylation for PET ligand discovery. Nature, doi:10.1038/s41586-020-3015-0 (2020).

33 McAtee, R. C., Noten, E. A. \& Stephenson, C. R. J. Arene dearomatization through a catalytic Ncentered radical cascade reaction. Nature 
Communications 11, 2528, doi:10.1038/s41467020-16369-4 (2020).

34 Chambers, R. C. \& Hill, C. L. Excited states of polyoxometalates as oxidatively resistant initiators of hydrocarbon autoxidation. Selective production of hydroperoxides. Inorganic Chemistry 28, 2509-2511, doi:10.1021/ic00312a002 (1989).

35 Tripathi, S., Singh, S. N. \& Yadav, L. D. S. Visible light photocatalysis with $\mathrm{CBr} 4$ : a highly selective aerobic photooxidation of methylarenes to aldehydes. RSC Advances 6, 14547-14551, doi:10.1039/C5RA26623H (2016).

36 Schultz, D. M. et al. Oxyfunctionalization of the Remote $\mathrm{C}-\mathrm{H}$ Bonds of Aliphatic Amines by Decatungstate Photocatalysis. Angewandte Chemie International Edition 56, 15274-15278, doi:https://doi.org/10.1002/anie.201707537 (2017).

37 Laudadio, G. et al. Selective C(sp3)-H Aerobic Oxidation Enabled by Decatungstate Photocatalysis in Flow. Angewandte Chemie International Edition 57, 4078-4082, doi:https://doi.org/10.1002/anie.201800818 (2018).

38 Lee, B. J., DeGlopper, K. S. \& Yoon, T. P. SiteSelective Alkoxylation of Benzylic $\mathrm{C}-\mathrm{H}$ Bonds by Photoredox Catalysis. Angewandte Chemie International Edition 59, 197-202, doi:10.1002/anie.201910602 (2020).

39 Tanwar, L., Börgel, J. \& Ritter, T. Synthesis of Benzylic Alcohols by C-H Oxidation. Journal of the American Chemical Society 141, 1798317988, doi:10.1021/jacs.9b09496 (2019).

40 Hu, A., Guo, J.-J., Pan, H. \& Zuo, Z. Selective functionalization of methane, ethane, and higher alkanes by cerium photocatalysis. Science 361, 668, doi:10.1126/science.aat9750 (2018).

41 An, Q. et al. Cerium-Catalyzed C-H Functionalizations of Alkanes Utilizing Alcohols as Hydrogen Atom Transfer Agents. Journal of the American Chemical Society 142, 6216-6226, doi:10.1021/jacs.0c00212 (2020).

42 Rivaton, A. \& Gardette, J.-L. Photooxidation of aromatic polymers. Angewandte Makromolekulare Chemie - ANGEW MAKROMOL CHEM 261, 173-188, doi:10.1002/(SICI)1522-9505(19981201)261262:13.3.CO;2-F (1998). 